\title{
Rank-Based Solution Methods and their Applications in Determination of Non-Dominated Points Set For A Multi-Objective Integer Programming Model
}

\author{
Ali Al-Hasani \\ School of Mathematical and Geospatial Sciences, RMIT University, Melbourne, Australia. \\ Department of Mathematics, College of Sciences, University of Basrah, Al- Basrah, Iraq. \\ E-mail: ali.alhasani@rmit.edu.au \\ Masar Al-Rabeeah \\ School of Mathematical and Geospatial Sciences, RMIT University, Melbourne, Australia. \\ Department of Mathematics, College of Sciences, University of Basrah, Al- Basrah, Iraq. \\ E-mail: masar.alrabeeah@rmit.edu.au \\ Santosh Kumar \\ School of Mathematical and Geospatial Sciences, RMIT University, Melbourne, Australia. \\ Department of Mathematics and Statistics, University of Melbourne, Melbourne, Australia. \\ Corresponding author: santosh.kumarau@gmail.com

\section{Andrew Eberhard} \\ School of Mathematical and Geospatial Sciences, \\ RMIT University, Melbourne, Australia. \\ E-mail: andy.eberhard@rmit.edu.au
}

(Received February 23, 2020; Accepted June 2, 2020)

\begin{abstract}
For any single-objective mathematical programming model, rank-based optimal solutions are computationally difficult to find compared to an optimal solution to the same single-objective mathematical programming model. In this paper, several methods have been presented to find these rank-based optimal solutions and based on them a new rank-based solution method (RBSM) is outlined to identify non-dominated points set of a multi-objective integer programming model. Each method is illustrated by a numerical example, and for each approach, we have discussed its limitations, advantages and computational complexity.
\end{abstract}

Keywords- Exact and approximate methods for ranked-optimal solutions; K-ranked optimal solutions; Multi-objective integer programming model; Non-dominated point set; Rank-based solution method.

\section{Introduction}

Although, single-objective mathematical programming models and their solution approaches have made a lot of contributions in solving many industrial and real-life optimization problems in operations research and other related areas of study, yet many of these situations are better represented by a multi-objective model, and therefore a need for solution approaches for these multi-objective models is ever increasing. An optimal solution for single-objective models has played a significant role in complex decision-making situations. In the case of multi-objective integer programming (MOIP) problems, instead of finding a single optimal solution, one is required to find the non-dominated points set, which is a central problem of interest. The optimal solution for a single-objective mathematical programming model is relatively easy, as the optimality of the 
International Journal of Mathematical, Engineering and Management Sciences

Vol. 5, No. 6, 1249-1269, 2020

https://doi.org/10.33889/IJMEMS.2020.5.6.093

solution is defined by some known conditions. These conditions help us to identify the optimal solution. However, the same is not true for the $K^{t h}$ ranked optimal solutions, $K \geq 2$.

The proposed rank-based solution method (RBSM) to identify the non-dominated point set of a given multi-objective integer programming model uses $K^{t h}$ ranked optimal solutions, $K \geq 2$. Thus, justifying a need for developing methods that can determine K-ranked optimal solutions for a single-objective mathematical programming model. In this paper, several methods have been discussed that can find these K-ranked optimal solutions and later a rank-based solution method has been developed for identifying the non-dominated point set of the given multi-objective integer programming model.

This paper has been organized into 9 Sections. Preliminaries are given in Section 2. Rank-based solution methods are discussed in Section 3. Literature concerning methods for determination of non-dominated point set has been reviewed in Section 4. The proposed rank-based solution method has been developed in Section 5. Some interesting observations about the ranked-optimal solutions have been stated in Section 6. The rank-based solution method for a multi-objective programming model is discussed in Section 7. Numerical illustrations, for the rank-based solution method discussed in this paper, are presented in Section 8. Finally, concluding remarks and future directions are presented in Section 9.

\section{Preliminaries}

The mathematical formulation of a single and multi-objective integer programming model is described as (1) and (2):

$\operatorname{MinZ}(x)$

$\operatorname{MinZ}(x)=\left(Z_{1}(x), Z_{2}(x), \ldots, Z_{p}(x)\right)$

Here (1) and (2) are subject to $x \in X$, where $X=\{A x \leq b ; x \geq 0$ and integer $\}$, which is the set of all feasible solution of (1) and (2) and $Z_{1}(x), Z_{2}(x), \ldots, Z_{p}(x)$ are the $p$ linear objective functions for the multi-objective problem. The elements of matrix $A$ are the coefficients of constraints, and $b$ is a vector of non-negative integer values. $X$ is called decision space and the image of $X(Y=Z(x))$ is called criterion or objective space. When $x \in\{0,1\}^{n}$ the problem is called a multi-objective combinatorial optimization problem. For this paper, the following notations are used.

Notation 2.1. The $K^{\text {th }}$ best optimal solution with respect to the objective $s$ is denoted by $Z_{s}^{K}, K=$ $1,2, \ldots$ are ranked optimal solutions and $s=1,2, \ldots, p$.

Notation 2.2. The value of the $l^{\text {th }}$ objective function, at the point $Z_{s}^{K}$ is denoted by $Z_{l @ Z_{S}^{K}}, l=$ $1,2, \ldots, p,(l \neq s)$.

Notation 2.3. Since $Z_{s}^{K}$ values may not be unique, let $r_{s}^{K}$ denote the number of such alternative solution $r_{s}^{K}=1,2, \ldots$.

Definition 2.4. The $K^{\text {th }}$ ranked optimal solution of $(1)$, denoted by $Z^{K}$ is the best solution in $W$, where

$$
W=\left\{\{A X \geq b, X \geq 0 \text { and integer }\} \cap\left(Z^{1}\right) \cap\left(Z^{2}\right) \cap \ldots \cap\left(Z^{(K-1)}\right)\right\}, Z^{1}=Z^{O p t} \text { and } K \geq 2
$$


International Journal of Mathematical, Engineering and Management Sciences

Vol. 5, No. 6, 1249-1269, 2020

https://doi.org/10.33889/IJMEMS.2020.5.6.093

The $K^{\text {th }}$ ranked optimal solution with respect to objective $Z_{s}$ in (2) will be,

$$
Z_{s}^{O p t} \leq Z_{s}^{2} \leq Z_{s}^{3} \leq \cdots \leq Z_{s}^{K}, \text { where } s=1,2, \ldots, p \text {, and } K \geq 1 .
$$

Definition 2.5. A feasible solution $x^{0} \in X$ is called efficient or (Pareto) solution, if there is no other $x \in X$ such that $Z\left(x^{0}\right) \geq Z(x)$. The set of all efficient solutions $x^{0} \in X$ denoted $X_{E}$ is called the efficient solution set.

Definition 2.6. (Dominance) Let $y^{1}$ and $y^{2}$ be two points in the objective space, $y^{1}$ dominates $y^{2}$, donated by $y^{1} \leq y^{2}$, if $y_{s}^{1} \leq y_{s}^{2}$ for $s=1,2, \ldots, p$.

Definition 2.7. If $x^{0}$ is efficient solution, $Z\left(x^{0}\right)$ is called non-dominated (Pareto) point (NDP), and the set of all non-dominated points $y^{0} \in Y$ is denoted $Y_{N D}$ and is called the non-dominated points set.

For further details see, Ehrgott (2005) and Steuer (1986). Various methods of integer programming have been reviewed in Kumar et al. (2010)

\section{Methods for Determination of Rank-Based Optimal Solutions}

Rank-based optimal solutions are difficult to identify as there are no defined conditions to identify the optimality of the $K^{\text {th }}$ best solution, $K \geq 2$. The $K^{\text {th }}$ best solution is defined as the best solution, excluding the $(K-1)$ best solutions. This requirement makes identification and determination of the $K^{\text {th }}$ best solution to a given integer programming model more difficult. Some methods for determination of rank-based optimal solution are presented in this section, which is broadly classified into two categories. Determination of the $K^{\text {th }}$ best solution, $K \geq 2$ for a linear integer program involving fractions are discussed in Section 3.1, and methods for unimodular models, which are free of fractional values are presented in Section 3.2.

\subsection{The Characteristic Equation Technique for Determination of Ranked-Optimal Solutions for A Single-Objective Linear Integer Program}

Kumar et al. (2007) obtained a linear relation from the objective row of the final simplex tableau of the LP relaxation of the given linear integer programming model as given in equation (3).

$Z+a_{m+1,1, s_{1}}+a_{m+1,2} s_{2}+\cdots+a_{m+1 . n} s_{n}=\mathrm{P}$

In equation (3), $s_{1}, s_{2}, \ldots, s_{n}$ are non-basic variables, $Z$ is the objective function, $a_{m+1, j}, j=$ $1,2, \ldots, n$ are the coefficients of these non-basic variables in the objective row and $\mathrm{P}$ denotes the value of the objective function. Usually, in the LP relaxation of the given pure integer linear programming model, the constants $a_{m+1, j}$ are fractional values. The value of the objective function is given by $\mathrm{P}$ under the condition that all non-basic variables at the optimal solution are zero. It may be noted that the values of $a_{m+1, j}$ are not fractional values for the unimodal problems. Note that equation (3) can be re-written as equation (4) given below.

$\left(\frac{D}{D}\right) Z+\frac{\alpha_{1} s_{1}+\alpha_{2} s_{2}+\cdots+\alpha_{n} s_{n}}{D}=\frac{P^{\prime}}{D}=\frac{R+i D}{D}$

In equation (4), $\mathrm{D}$ is the common denominator, $\mathrm{R}$ is the integer residue of $\left(\frac{P^{\prime}}{D}\right)$ and $\alpha_{j}$ represents 
International Journal of Mathematical, Engineering and Management Sciences

Vol. 5, No. 6, 1249-1269, 2020

https://doi.org/10.33889/IJMEMS.2020.5.6.093

the integer coefficient of the non-basic variable $s_{j}$ for $j=1,2, \ldots, n$. Note that $a_{m+1, j}=\frac{\alpha_{j}}{D}$.

From (4), Kumar et al. (2007) obtained a linear relation (5) and called it a descending hyperplane.

$\sum_{j=1}^{n} \alpha_{j} s_{j}=R+i D, i=0,1,2, \ldots,\left\lceil\frac{P^{\prime}}{D}\right\rceil$

Equation (5) was later renamed by Kumar and Munapo (2012) as the characteristic equation for a pure integer programming model. Note that (5) is a necessary condition but it is not enough to ensure integer solutions. For integer solutions, the slack variables at the integer point need not be zero as in a linear programming model. However, when they are not zero, they also will be restricted to integer values. The LHS is a sum of these non-basic integer variables, which must be equal to $R+i D$. The efficiency of the relation (5) in the identification of integer points is a function of these three parameters on the RHS i.e. the values $R, D$ and $i$. The largest value of $i$ is governed by the highest integer value of $\left\lceil\frac{P^{\prime}}{D}\right\rceil$.

Equation (5) has interesting characteristics. For example:

- A linear relation (5) will have positive $\mathrm{R}$ and $\mathrm{D}$ values $\geq 1$, only when the objective function row of LP relaxation involves fractional values.

- In the case of unimodal functions $R=0, D=1$ and $\left\lceil\frac{P^{\prime}}{D}\right\rceil=p^{\prime}$, hence the CE approach is not suitable for a unimodular function. Other methods for unimodular problems are required and they are discussed in section 3.2.

- The solutions obtained with the help of relation (5) are ranked-optimal solutions in descending order. This is a unique feature of the CE, which is not seen in any other optimization method. Thus, the CE provides the optimal solution, as well as other ranked-optimal solutions in descending order.

\section{Numerical Illustration 1}

Consider a trivial 2-variable problem taken from Rabeeah et al. (2019).

$z=x_{1}-x_{2}$, subject to: $x_{1}+6 x_{2} \leq 21,14 x_{1}+6 x_{2} \leq 63, x_{1}, x_{2} \geq 0$ and integer

The optimal solution of the LP relaxation of (6) is given in Table 1. From the objective row in Table 1, the $\mathrm{CE}$ will be as given by (7):

$20 x_{2}+s_{2}=7+14 i, i=0,1,2,3$ and 4 as $\left\lceil\frac{63}{14}\right\rceil=4$.

Analysing equation (7) for $i=0$, equation (7) becomes $20 x_{2}+s_{2}=7$, which has a feasible solution $s_{2}=7$. For this value of the non-basic variable, the basic variables

with the help of Table 1 becomes: 
International Journal of Mathematical, Engineering and Management Sciences

Vol. 5, No. 6, 1249-1269, 2020

https://doi.org/10.33889/IJMEMS.2020.5.6.093

Table 1. Optimal LP solution

\begin{tabular}{|c|c|c|c|c|c|}
\hline Basic & $x_{1}$ & $x_{2}$ & $s_{1}$ & $s_{2}$ & RHS \\
\hline$s_{1}$ & 0 & $78 / 14$ & 1 & $-1 / 14$ & $231 / 14$ \\
\hline$x_{1}$ & 1 & $6 / 14$ & 0 & $1 / 14$ & $63 / 14$ \\
\hline$z_{j}-c_{j}$ & 0 & $20 / 14$ & 0 & $1 / 14$ & $63 / 14$ \\
\hline
\end{tabular}

$$
\begin{aligned}
& s_{1}=\frac{231}{14}+7\left(\frac{1}{14}\right)-\left(\frac{78}{14}\right)(0)=17 . \\
& x_{1}=\left(\frac{63}{14}\right)-7\left(\frac{1}{14}\right)-\left(\frac{6}{14}\right)(0)=4 .
\end{aligned}
$$

Since $x_{1}=4, x_{2}=0, s_{1}=17$ and $s_{2}=7$ is a feasible integer solution, it is the required optimal solution with $z=4$.

For the $2^{\text {nd }}$ best solution, returning to the equation (7) with $i=1$, resulting in

$$
20 x_{2}+s_{2}=21 \text {. }
$$

The above equation has two alternative solutions $x_{2}=s_{2}=1$ and $x_{2}=0, s_{2}=21$. Note the first solution will result into $x_{1}=4, x_{2}=1, s_{1}=11$ and $s_{2}=1$, giving $z=3$. The second solution gives $x_{1}=3, x_{2}=0, s_{1}=18$ and $s_{2}=21$, giving $z=3$. Thus we have two $2^{\text {nd }}$ best solutions.

Once again for the $3^{\text {rd }}$ best solution, substitute $i=2$, resulting in

$$
20 x_{2}+s_{2}=35 \text {. }
$$

The above equation has again two possible solutions. They are: $x_{2}=1$ and $s_{2}=15$ and $x_{2}=$ $0, s_{2}=35$. The first solution results in $x_{1}=3, x_{2}=1, s_{1}=12$ and $s_{2}=15$, giving $z=2$. The second solution results in $x_{1}=2, x_{2}=0, s_{1}=19$ and $s_{2}=35$, giving $z=2$. Thus we have two alternative $3^{\text {rd }}$ best solutions.

Similarly, one can trace other solutions and verify them graphically.

\subsection{Determination of Rank-Based Optimal Solutions for Unimodular Models}

The CE approach is not applicable for the unimodular models, such as assignment and transportation models. For ease of presentation, we discuss the case of an assignment model and find the $K^{\text {th }}$ best solution by the Random Search Method developed by Kumar et al. (2018). It is presented in section 3.2.1.

\subsubsection{A Random Search Method to Find the Rank-Based Solutions of An Assignment Model}

The random search method is a probabilistic approach to find the $K^{\text {th }}$ best solution of a unimodular model, such as an assignment model. We find the $K^{\text {th }}$ best solution for a given assignment model, $K \geq 2$. The RSM can be described by the following steps:

- One is required to find the number of possibilities for estimating the probability of a successful 
International Journal of Mathematical, Engineering and Management Sciences

Vol. 5, No. 6, 1249-1269, 2020

https://doi.org/10.33889/IJMEMS.2020.5.6.093

solution in the feasible domain. In an assignment problem of size $n \times n$, if the optimal solution is unique and after determination of that optimal solution, one has $(n(n-1))$ unoccupied cells. These unoccupied cells offer possibilities of other ranked solutions. However, if there $r_{1}$ number of alternative optimal solutions, the possibilities become $r_{1}(n(n-1))$. Therefore, the probability of a successful solution is given by: $=\frac{1}{r_{1}(n(n-1))}, r_{1} \geq 1$. .

Here a successful solution means that a solution picked up randomly may be the required $K^{\text {th }}$ best solution with probability $p=\frac{1}{r_{1}(n(n-1))}$. In general, for the $K^{\text {th }}$ best solution, $K \geq 2$, the probability will be given by: $p=\frac{1}{n(n-1) \sum_{i=1}^{i=(K-1)} r_{i}}, r_{i} \geq 1, i=1,2, \ldots,(K-1)$.

- The value of assurance factor, denoted by (PR), is a given probability assigned for assurance of the results we find by the computational experiments. Here we have assumed $P R=0.85$.

- In the application of RSM one needs the rule to stop these random searches, which is given by relation (8), where (NS) denotes the number of random searches.

$$
N S=\frac{\ln (1-P R)}{\ln \left(1-\frac{1}{n(n-1) \sum_{i=1}^{K-1} r_{i}}\right)}
$$

This random search approach has been executed as Algorithm 1.

\section{Algorithm 1 (Random Search Method for Finding "Kth" Best Optimal Solution)}

Step 1. Initialize

$1.1 \quad$ Set $Z_{\text {ranked }} \leftarrow-Z_{t}^{\text {opt }} ; t=1,2, . . r_{1} \quad$ ( $r_{1}$ number of multi optimal solutions)

1.2 Let $L \geq 2$ and integer ( $L$ number of ranked optimal solution required)

$1.3 p=\frac{1}{r_{1}(n(n-1))} ; 0 \leq P R \leq 1$ ( $p$ is probability; $P R$ is the value of assurance factor)

$1.4 S=0 ; K=2(S$ is counter of $Z ; K$ is second best optimal solution $)$

\section{Step 2 .}

$$
\begin{aligned}
& 2.1 \quad Z^{*} \leftarrow-\emptyset . \quad \text { ( } Z^{*} \text { is a set of } Z_{S} \text { values) } \\
& 2.2 \quad N S=\frac{\ln (1-P R)}{\ln \left(1-\frac{1}{n(n-1) \sum_{i=1}^{K-1} r_{i}}\right)} \\
& 2.3 X_{\text {rand }} \leftarrow-\emptyset\left(X_{\text {rand }} \text { is a set of } x_{i j}\right) \text {. }
\end{aligned}
$$

\section{Step 3. Main Loop}

3.1 Do $S=S+1$

3.2 Choose $\operatorname{rand}\left(x_{i j}\right)_{S}$.

3.3 If $x_{i j} \in X_{\text {rand }}$ then ignore $\operatorname{rand}\left(x_{i j}\right)_{S}$ GoTo Step 3.2, else continue

3.4 Add $x i j \rightarrow X$ rand.

3.5 Rand $\left(x_{i j}\right)_{s} \Rightarrow x_{i j}^{*}, \quad x_{i j}^{*} \quad$ is optimal solution) 
International Journal of Mathematical, Engineering and Management Sciences

Vol. 5, No. 6, 1249-1269, 2020

https://doi.org/10.33889/IJMEMS.2020.5.6.093

3.6 $\quad Z^{*} \leftarrow-Z_{S} . \quad\left(Z_{S}\right.$ solution at $\left.\operatorname{rand}\left(x_{i j}\right)_{S}\right)$

3.73 .7 If $S=(N S+1)$ GoTo 4. Else GoTo Step 3 .

Step 4. Rank $Z^{*} \rightarrow$ find the best $Z^{K} ; Z_{\text {ranked }} \leftarrow-Z^{K t h}$.

Step 5. If $K=L$ GoTo Step 6 . Else $K=K+1$ Goto Step 2 .

Step 6. Rank $Z_{\text {ranked }}$

\section{Numerical Illustration 2}

We consider an assignment problem (9)

$\operatorname{Min} \mathrm{Z}=\sum_{i=1}^{4} \sum_{j=1}^{4} C_{i j} x_{i j}$

Subject to

$$
\begin{aligned}
& \sum_{i=1}^{4} x_{i j}=1 ; j=1,2, \ldots, 4 \\
& \sum_{j=1}^{4} x_{i j}=1 ; i=1,2, \ldots, 4
\end{aligned}
$$

Such that: $C_{i j}=\left[\begin{array}{llll}7 & 5 & 5 & 9 \\ 2 & 9 & 9 & 7 \\ 4 & 2 & 7 & 1 \\ 2 & 1 & 5 & 7\end{array}\right]$

We select the $\mathrm{PR}=0.80, ; \mathrm{K}=2$ and we find $p=\frac{1}{(K-1) n(n-1)}=\frac{1}{12}$.

Then, the number of search (NS) from the equation:

$N S=\left[\frac{\ln (1-P R)}{\ln (1-p)}\right]=\left[\frac{\ln (1-0.8)}{\ln \left(1-\frac{1}{12}\right)}\right]=18 ;[\quad]$ is nearest integer function

Step 1.

The optimal solution of the assignment problem is:

\begin{tabular}{|c|c|c|c|c|}
\hline & $\mathrm{J} 1$ & $\mathrm{~J} 2$ & $\mathrm{J3}$ & $\mathrm{J} 4$ \\
\hline $\mathrm{S} 1$ & 2 & 0 & 0 & 4 \\
\hline $\mathrm{S} 2$ & 0 & 7 & 7 & 5 \\
\hline $\mathrm{S3}$ & 3 & 1 & 6 & 0 \\
\hline $\mathrm{S} 4$ & 1 & 0 & 4 & 6 \\
\hline
\end{tabular}

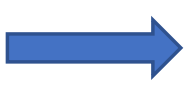

\begin{tabular}{|c|c|c|c|c|}
\hline & $\mathrm{J} 1$ & $\mathrm{~J} 2$ & $\mathrm{J3}$ & $\mathrm{J} 4$ \\
\hline $\mathrm{S} 1$ & $z$ & 0 & 0 & 4 \\
\hline $\mathrm{S} 2$ & 0 & 7 & 7 & 5 \\
\hline $\mathrm{S3}$ & 3 & 1 & 6 & 0 \\
\hline $\mathrm{S} 4$ & 1 & 0 & 4 & 6 \\
\hline
\end{tabular}


International Journal of Mathematical, Engineering and Management Sciences

Vol. 5, No. 6, 1249-1269, 2020

https://doi.org/10.33889/IJMEMS.2020.5.6.093

Step 2.

The number of searches, NS is equal to 18. Choose randomly a non-basic cell and let that cell be $x_{11}$. Then the non-basic cells $x_{11}$ and $x_{23}$ will be replaced with basic cells $x_{13}$ and $x_{21}$.

\begin{tabular}{|l|l|l|l|l|}
\hline & J1 & J2 & J3 & J4 \\
\hline S1 & 2 & 0 & 0 & 4 \\
\hline S2 & 0 & 7 & 7 & 5 \\
\hline S3 & 3 & 1 & 6 & 0 \\
\hline S4 & 1 & 0 & 4 & 6 \\
\hline
\end{tabular}

The solution and $\mathrm{Z}$ from the above table is: $X=x_{11}, x_{23}, x_{34}, x_{42}, Z_{1,2}=18$.

We continue this process NS number of times to compute $Z_{1,3}, Z_{1,4}, \ldots, Z_{1,18}$.

Step 3.

These solutions are arranged as: $Z_{1,2}, Z_{1,3}, Z_{1,4}, \ldots, Z_{1,18}$ in descending order. Many solutions are repeated, thus from the 18 randomly generated solutions, we obtain only 6 distinct solutions as given in $\mathrm{Z}$ value of Table 2 .

Table 2. Results from the random search

\begin{tabular}{|c|c|}
\hline $\mathrm{Z}$ & $\mathrm{X}$ \\
\hline $\mathrm{Z}^{*}=9$ & $x_{13}, x_{21}, x_{34}, x_{42}$ \\
\hline$Z_{13}=13$ & $x_{12}, x_{21}, x_{34}, x_{43}$, \\
\hline$Z_{15}=16$ & $x_{13}, x_{22}, x_{32}, x_{44}$, \\
\hline$Z_{14}=17$ & $x_{13}, x_{24}, x_{31}, x_{42}$, \\
\hline$Z_{17}=17$ & $x_{13}, x_{22}, x_{34}, x_{41}$, \\
\hline$Z_{16}=18$ & $x_{11}, x_{23}, x_{34}, x_{42}$, \\
\hline$Z_{12}=19$ & $x_{14}, x_{21}, x_{33}, x_{42}$, \\
\hline
\end{tabular}

From Table2, we take $Z_{13}$ representing the 2 nd-best solution with probability 0.80 .

Step 4.

We choose from Table 2 , the solution $Z_{13}$, then reapply step 2 and step 3 on the selected solution. This process is continued for $\mathrm{K}=5$ ranked-best solutions, which is shown in Table 3 
International Journal of Mathematical, Engineering and Management Sciences

Vol. 5, No. 6, 1249-1269, 2020

https://doi.org/10.33889/IJMEMS.2020.5.6.093

Table $3 . Z^{5^{t h}}$ best assignment solution for illustrative example

\begin{tabular}{|c|c|c|c|}
\hline$Z^{K}$ & $\mathrm{Z}$ value & $X$ & Alternative solutions \\
\hline$Z^{1}$ & 9 & $x_{13}, x_{21}, x_{34}, x_{42}$ & 1 \\
\hline$Z^{2 n d}$ & 13 & $x_{12}, x_{21}, x_{34}, x_{43}$ & 1 \\
\hline \multirow[t]{2}{*}{$Z^{3 d r}$} & \multirow[t]{2}{*}{16} & $x_{13}, x_{21}, x_{32}, x_{44}$ & \multirow[t]{2}{*}{2} \\
\hline & & $x_{13}, x_{24}, x_{32}, x_{41}$ & \\
\hline \multirow[t]{3}{*}{$Z^{4^{t h}}$} & \multirow[t]{3}{*}{17} & $x_{13}, x_{24}, x_{31}, x_{42}$ & \multirow[t]{3}{*}{3} \\
\hline & & $x_{13}, x_{22}, x_{34}, x_{41}$ & \\
\hline & & $x_{12}, x_{23}, x_{34}, x_{41}$ & \\
\hline$Z^{5^{t h}}$ & 18 & $x_{14}, x_{21}, x_{32}, x_{43}$ & 1 \\
\hline
\end{tabular}

\subsubsection{Murty's Approach for Ranked-Based Solutions}

Murty (1968) suggested to solve the given $n X n$ assignment model by the Hungarian method and let the optimal solution be denoted by a string of $n$ allocations satisfying the assignment constraints. Let the string representing the optimal solution be denoted by

$a(1)=\left\{\left(i_{1}, j_{1}\right),\left(i_{2}, j_{2}\right), \ldots,\left(i_{n}, j_{n}\right)\right\}$. Let the total cost associated with this optimal solution be denoted by TC(1). Since the $2^{\text {nd }}$ best solution will differ from the best by at least one allocation, Murty solved $n-1$ more problems by substituting, one at a time $\left(i_{l}, j_{l}\right)=\infty, l=1,2, \ldots, n-1$. Let these total costs be denoted by $T C\left(\left(i_{1}, j_{1}\right)=\infty\right), T C\left(\left(i_{2}, j_{2}\right)=\infty\right), \ldots, T C\left(\left(i_{n-1}, j_{n-1}\right)=\infty\right)$.

Minimum total cost for these $n-1$ problems will be the required $2^{\text {nd }}$ best solution and the allocation will be represented by its corresponding string and the cost matrix will be denoted with appropriate link, $\left(i_{l}, j_{l}\right)=\infty$. The process will continue for the subsequent $K^{\text {th }}$ best solution, $K \geq 2$. Thus, for every $\mathrm{K}$, one has to solve at least $n-1$ more problems of size $n X n, \ldots, 2 X 2$, with one or more elements equal to $\infty$ in the given cost matrix.

\section{Numerical Illustration 3}

Murty's approach is illustrated, using the same numerical illustration 2, discussed above.

Step 1.

The optimal solution of the assignment problem (9) is:

\begin{tabular}{|c|c|c|c|c|}
\hline & J1 & J2 & J3 & J4 \\
\hline S1 & 2 & 0 & 0 & 4 \\
\hline S2 & 0 & 7 & 7 & 5 \\
\hline S3 & 3 & 1 & 6 & 0 \\
\hline S4 & 1 & 0 & 4 & 6 \\
\hline
\end{tabular}


International Journal of Mathematical, Engineering and Management Sciences

Vol. 5, No. 6, 1249-1269, 2020

https://doi.org/10.33889/IJMEMS.2020.5.6.093

Step 2.

Return back to problem (9) and set one basic cell equal to a large number, say 1000, which are the basic cells i.e., $(1,3),(2,1),(3,4)$ and $(4,2)$, therefore 4 new assignment problems will have to be solved. The first one will be as shown below.

\begin{tabular}{|c|c|c|c|c|}
\hline & J1 & J2 & J3 & J4 \\
\hline S1 & 7 & 5 & 1000 & 9 \\
\hline S2 & 2 & 9 & 9 & 7 \\
\hline S3 & 4 & 2 & 7 & 1 \\
\hline S4 & 2 & 1 & 5 & 7 \\
\hline
\end{tabular}

The optimal solution for the assignment data with cell $(1,3)=1000$ is given by:

Optimal cost $=13$, Optimal allocation is at cells $(1,2),(2,1),(3,4)$ and $(4,3)$. Similarly, results of the remaining three problems are shown in Table 4.

Table 4. Finding the second best the optimal solution using Murty's approach

\begin{tabular}{|l|c|c|}
\hline Basic cell $=1000$ & Z value & $X$ \\
\hline$X_{13}$ & 13 & $x_{12}, x_{21}, x_{34}, x_{43}$ \\
\hline$X_{21}$ & 16 & $x_{13}, x_{24}, x_{32}, x_{41}$ \\
\hline$X_{34}$ & 16 & $x_{13}, x_{21}, x_{32}, x_{44}$ \\
\hline$X_{42}$ & 13 & $x_{12}, x_{21}, x_{34}, x_{43}$ \\
\hline
\end{tabular}

Therefore, the second-best solution is 13 , and optimal allocation is $\left\{x_{12}, x_{2} 1, x_{34}, x_{43}\right\}$. The similar analysis will have to be carried out for the $3^{\text {rd }}$ best solution from the second-best solution. However, it may be noted that apparently the second-best solution is resulting in the same allocation from two different cases. Therefore, we must solve 8 problems, four for each case. Four problems will be solved with cell $(1,3)=1000$ and one at a time i.e. cell $(1,2),(2,1),(3,4)$ and $(4,3)=1000$ and other four problems will be with the cell $(4,2)=1000$ and one at a time cell $(1,2),(2,1),(3,4)$ and $(4,3)$. Murthy does not consider this possibility.

\section{Literature Review for Non-Dominated Point Set for a Multi-Objective Integer Programming Model}

There are many approaches in the literature that have been developed to find the non-dominated points set, for a given multi-objective integer programming (MOIP) model, for example see, (Steuer, 1986; Ehrgott, 2005; Greco et al., 2016). Multi-objective combinatorial optimization (MOCO) problems are a special case of MOIP problems, where all variables are binary. For 
International Journal of Mathematical, Engineering and Management Sciences

Vol. 5, No. 6, 1249-1269, 2020

https://doi.org/10.33889/IJMEMS.2020.5.6.093

example, see the survey by Ehrgott and Gandibleux (2000), which deals with a variety of methods and models for MOCO problems. They have discussed some exact and approximate methods for the identification of non-dominated point set for MOIP models. Ulungu and Teghem (1995) have presented a two phases method (TPM) to find all non-dominated points for bi-objective assignment problems. In phase one, the supported non-dominated points are determined. In phase two, the nonsupported points are obtained. The TPM was improved by Przybylski et al. (2008) and they applied to solve the three-objective assignment problem (Przybylski et al., 2010b). TPM has also been applied on several other MOCO models Al-Hasani et al. (2019). Sylva and Crema $(2004,2007)$ have provided algorithms for generating non-dominated points set with limited success, particularly for larger size instances. Özlen and Azizoğlu (2009) developed an approach based on the $\epsilon$ - constraint method, which was developed by Haimes (1971). Przybylski et al. (2010a) have presented an algorithm that generates all non-dominated points for MOIP problems; they tested their algorithm on three objective assignments and knapsack problems. The well-established $\varepsilon$ constraint scalarization and partitioning mechanism were used by Kirlik and Sayin (2014) and compared with Özlen and Azizoğlu (2009). Özlen et al. (2014) modified their previous approach and presented a comparison with their previous work for MOA problems. Boland et al. (2015) have presented a new approach, called Balanced Box Method (BBM) for bi-objective integer programming models. The BBM solves IP's three times the number of non-dominated points and therefore, it takes a lot of time to generate the non-dominated set. The $L$-shape search method has been presented by Boland et al. (2016) and applied to three-objective integer programmes and compared with Özlen et al. (2014) and Kirlik and Sayın (2014). Al-Rabeeah et al. (2020) developed an improved recursive method that out preformed many existing methods, mainly due to adopting a different scalarization scheme. This method decreased the number of sub-problems solved and hence the related CPU time was improved accordingly.

The complexity of a multi-objective assignment problem has been described by many authors, for example, see Ehrgott (2005). Due to these complexities and difficulties, the researchers have justified a need for approximation approaches to find the non-dominated points set. One such approach is by Teghem et al. (2000), which generalizes the multi-objective simulated annealing (MOSA) method. An interactive approach to generate the set of non-dominated points was developed by Tuyttens et al. (2000). Laumanns et al. (2006) presented a method to generate an approximate set of non-dominated point set for the MOA problem.

In this paper, a new approach to generate the non-dominated points set for a multi-objective integer programming (MOIP) model has been developed. The method is based on identifying the ranked optimal solutions to a single-objective integer programming model. As seen earlier in three numerical illustrations, unlike the optimal solution of a single-objective model, the ranked optimal solutions are computationally more demanding, as they do not have defined conditions for optimality of the $K^{\text {th }}$ best solution. Several methods have been discussed in this paper and developing more methods is a future challenge for further investigation.

Although computationally these methods are demanding but are easy to understand, and implement with in terms of CPU time and number of IP's is much less compared to many previous methods such as Balanced Box method by Boland et al. (2015), MOIP-AIRA by Özlen et al. (2014) etc. 
International Journal of Mathematical, Engineering and Management Sciences

Vol. 5, No. 6, 1249-1269, 2020

https://doi.org/10.33889/IJMEMS.2020.5.6.093

\section{Rank Based Solution Method for Determination of the Non-Dominated Point Set for a Bi-Objective Linear Integer Programming Model}

The RBSM method for Bi-objective linear integer programming (BOIP) model comprised of the following steps:

Step 1. Find the upper bound $Z_{U B}=\left(Z_{1}^{1}, Z_{2 @ Z_{1}^{1}}\right)$ and the lower bound $\left(Z_{L B}\right)=\left(Z_{1 @ Z_{2}^{1}}, Z_{2}^{1}\right)$, which are the initial two non-dominated points for the given bi-objective problem. Here, $Z_{1}^{1}=Z_{1}^{o p t}$ and, $Z_{2}^{1}=Z_{2}^{o p t}$ respectively.The $\left(Z_{U B}\right)$ and $\left(Z_{L B}\right)$ determines the endpoint of the search for the nondominated points set.

Step 2. Find the ranked optimal solution for $Z_{1}$ and $Z_{2}$ by using an appropriate method and denote them by $\left\{Z_{1}^{1}, Z_{1}^{2}, \ldots, Z_{1}^{K}\right\}$ and $\left\{Z_{2}^{1}, Z_{2}^{2}, \ldots, Z_{2}^{K}\right\}$, respectively. The value of $K$ may be different for the two objectives, as $Z_{1}^{1} \leq Z_{1}^{K} \leq Z_{1 @ Z_{2}^{1}}$ and $Z_{2}^{1} \leq Z_{2}^{K} \leq Z_{2 @ Z_{1}^{1}}$.

Step 3. Find the potential non-dominated points with respect to $Z_{1}$ and $Z_{2}$ and denoted by

$\left(Z_{1 @ Z_{2}^{l_{2}}}, Z_{2}^{l_{2}}\right) ; l_{2}=2,3, \ldots, K$ and $\left(Z_{1}^{l_{1}}, Z_{2 @ z_{1}^{l_{1}}}\right) ; l_{1}=2,3, \ldots, K$, respectively.

Step 4. The non-dominated point set $Y_{N D}$ is a subset of potential non-dominated point set which is obtained by testing each potential non-dominated point for dominance.

The Algorithm 2 provide the stepwise description of RBSM for BOLIP model.

Algorithm 2. (RBSM Algorithm for BOIP Problem)

Let $\mathrm{Z}$ Bi-objective linear integer programming model

\section{Initialize}

i. $\operatorname{RBS}\left(Z_{1}\right) \leftarrow \varnothing ; \operatorname{RBS}\left(Z_{2}\right) \leftarrow \emptyset ; \operatorname{PNDP} \leftarrow \emptyset ; \mathrm{NDP} \leftarrow \varnothing$

ii. $\quad \mathrm{NDP} \leftarrow \mathrm{Z}_{\mathrm{UP}}=\left(\mathrm{Z}_{1 \mathrm{opt}}, \mathrm{Z}_{2} @ \mathrm{Z}_{1 \mathrm{opt}}\right) \& \mathrm{Z}_{\mathrm{LR}}=\left(\mathrm{Z}_{1} @ \mathrm{Z}_{2 \mathrm{opt}}, \mathrm{Z}_{2 \mathrm{opt}}\right)$

$$
\begin{aligned}
& \operatorname{RBS}\left(Z_{1}\right) \leftarrow Z_{1}^{2}, \ldots, Z_{1 @ Z_{2}^{o p t}} \\
& \operatorname{RBS}\left(Z_{2}\right) \leftarrow\left\{Z_{2}^{2}, \ldots, Z_{2 @ Z_{1}^{o p t}}\right\}
\end{aligned}
$$

iii. Find PNDP points for RBS(Z1) and Add $\rightarrow$ PNDP

Find PNDP points for RBS(Z2) and Add $\rightarrow$ PNDP;

iv. If PNDP point is non-dominated point $\rightarrow$ NDP; else $\rightarrow$ delete PNDP point.

v. Stop.

\section{Some Interesting Observations about the Ranked Based Solutions}

The Rank Based Solution Method finds the non-dominated points set $Y_{N D}$ for the given MOIP model. It first finds ordered integer optimal solutions with respect to an objective $Z_{s}$, where $s=$ $1,2, . . p$.

Observation 6.1. A unique optimal solution with respect to the objective $\mathrm{Z}_{\mathrm{s}}$ is a non-dominated 
International Journal of Mathematical, Engineering and Management Sciences

Vol. 5, No. 6, 1249-1269, 2020

https://doi.org/10.33889/IJMEMS.2020.5.6.093

point for the given MOIP. In addition, the ranked optimal solutions with respect to each objective $\mathrm{Z}_{\mathrm{s}}$ are also potential non-dominated points for the given MOIP.

Observation 6.2. The relationship between the ranked best solutions for a single objective function $\mathrm{Z}_{\mathrm{s}}$ and non-dominated point set $\mathrm{Y}_{\mathrm{ND}}$ in the criterion space is very strong. All non-dominated points are ranked optimal solutions, but all ranked optimal solutions are not necessarily points of the non-dominated point set.

Observation 6.3. A unique optimal solution for a single objective integer programming model has a property that it always leads to an upper bound or a lower bound of the non-dominated point set for the bi-objective MOIP model. However, for the multi-optimal solutions, one and only one of them will result in an upper bound or a lower bound point of the non-dominated point set.

Observation 6.4. A single objective integer programming model may have $r_{s}^{K} \geq 1$, number of multi ranked optimal solutions for a given objective $s, s=1,2, \ldots, p$. From these $r_{s}^{K}$ number of alternative solutions, one and only one solution will become potential non-dominated point in the objective space.

\section{Ranked Based Solution Method for Multi-Objective Integer Programming Model}

The Rank Based Solution Method (RBSM) discussed above for the bi-objective integer model can be easily extended for determination of the non-dominated points set $Y_{N D}$ for a given multi-objective integer programming model. Once again, the approach is based on the determination of ordered optimal solutions with respect to an objective $Z_{s}$, where $s=1,2, \ldots, p$. and each point is tested for dominance.

\section{Algorithm 3. (RBSM Algorithm for MOIP Problem)}

Let Z Bi-Objective Linear Integer Programming Model

$$
\begin{aligned}
& \text { Initialize } \\
& \text { i. } \quad \operatorname{RBS}\left(Z_{1}\right) \leftarrow \varnothing ; \operatorname{RBS}\left(Z_{2}\right) \leftarrow \emptyset ; \operatorname{PNDP} \leftarrow \emptyset ; \mathrm{NDP} \leftarrow \varnothing \\
& \text { ii. } \quad \mathrm{NDP} \leftarrow \mathrm{Z}_{\mathrm{UP}}=\left(\mathrm{Z}_{\text {lopt }}, \mathrm{Z}_{2} @ \mathrm{Z}_{\text {lopt }}\right) \& \mathrm{Z}_{\mathrm{LR}}=\left(\mathrm{Z}_{1} @ \mathrm{Z}_{2 \mathrm{opt}}, \mathrm{Z}_{\text {2opt }}\right) \\
& \operatorname{RBS}\left(Z_{1}\right) \leftarrow Z_{1}^{2}, \ldots, Z_{1 @ Z_{2}^{o p t}} \\
& \operatorname{RBS}\left(Z_{2}\right) \leftarrow\left\{Z_{2}^{2}, \ldots, Z_{2 @ z_{1}^{o p t}}\right\}
\end{aligned}
$$

\section{Computational Illustration of the Rank-Based Solution Method}

In this section, two numerical examples are presented to illustrate the bi-objective and multiobjective problem for the application of the rank-based solution method. 
International Journal of Mathematical, Engineering and Management Sciences

Vol. 5, No. 6, 1249-1269, 2020

https://doi.org/10.33889/IJMEMS.2020.5.6.093

\subsection{Numerical Illustrations}

\section{Example 4}

Consider a BOAM instance of size $4 \times 4$.

Subject to

$$
\operatorname{MinZ}(x)=\sum_{i=1}^{4} \sum_{j=1}^{4} P_{i j}^{r} x_{i j} r=1,2
$$

where,

$$
\begin{gathered}
\sum_{j=1}^{4} x_{i, j}=1 \quad i=1, \ldots, 4 . \\
\sum_{i=1}^{4} x_{i, j}=1 \quad j=1, \ldots, 4 . \\
x_{i, j}=\left\{\begin{array}{l}
0 \\
1
\end{array} ; \forall(i, j) .\right.
\end{gathered}
$$

$$
P_{i, j}^{1}=\left[\begin{array}{llll}
5 & 2 & 9 & 6 \\
1 & 10 & 8 & 2 \\
2 & 3 & 8 & 9 \\
5 & 7 & 3 & 1
\end{array}\right], P_{i, j}^{2}=\left[\begin{array}{llll}
2 & 20 & 1 & 12 \\
10 & 4 & 2 & 13 \\
22 & 5 & 4 & 1 \\
2 & 4 & 32 & 11
\end{array}\right]
$$

- Find the optimal solution in respect of $Z_{1}$ and $Z_{2}$. Let the optimal be denoted by $Z_{1}^{1}, Z_{2}^{1}$, respectively. The value of $Z_{1}^{1}=9$ and $Z_{2}^{1}=8$.

- Find the value of $Z_{2}$ at the point where $Z_{1}^{1}=9$. This gives an upper non-dominated point $\left(Z_{1}^{1}, Z_{2 @ Z_{1}^{1}}\right)=(9,87)$. Similarly, the lower non-dominated point $\left(Z_{1 @ Z_{2}^{1}}, Z_{2}^{1}\right)=(33,8)$.

- Using the RSM find ranked optimal solutions in respect of $Z_{1}$, denoted by $Z_{1}^{K}, K=2,3, \ldots$, such that $Z_{1}^{K} \leq 33$. Similarly, repeat on $Z_{2}$, such that $Z_{2}^{K} \leq 87, K=2,3, \ldots$.

- $\quad$ For each $Z_{1}^{K}$, find $Z_{2 @ Z_{1}^{K}}$ to obtain a potential non-dominated point denoted by $\left(Z_{1}^{K}, Z_{2 @ Z_{1}^{K}}\right)$, see Table 5. Similarly, determine the potential non-dominated point in respect of $Z_{2}^{K}$, which are denoted $\left(Z_{1 @ Z_{2}^{K}}, Z_{2}^{K}\right)$, see Table 6.

- Using definition 6, find the non-dominated set from all potential non-dominated points as shown in Figure 1.

- All Non- dominated point are also shown in Table 7. 
International Journal of Mathematical, Engineering and Management Sciences

Vol. 5, No. 6, 1249-1269, 2020

https://doi.org/10.33889/IJMEMS.2020.5.6.093

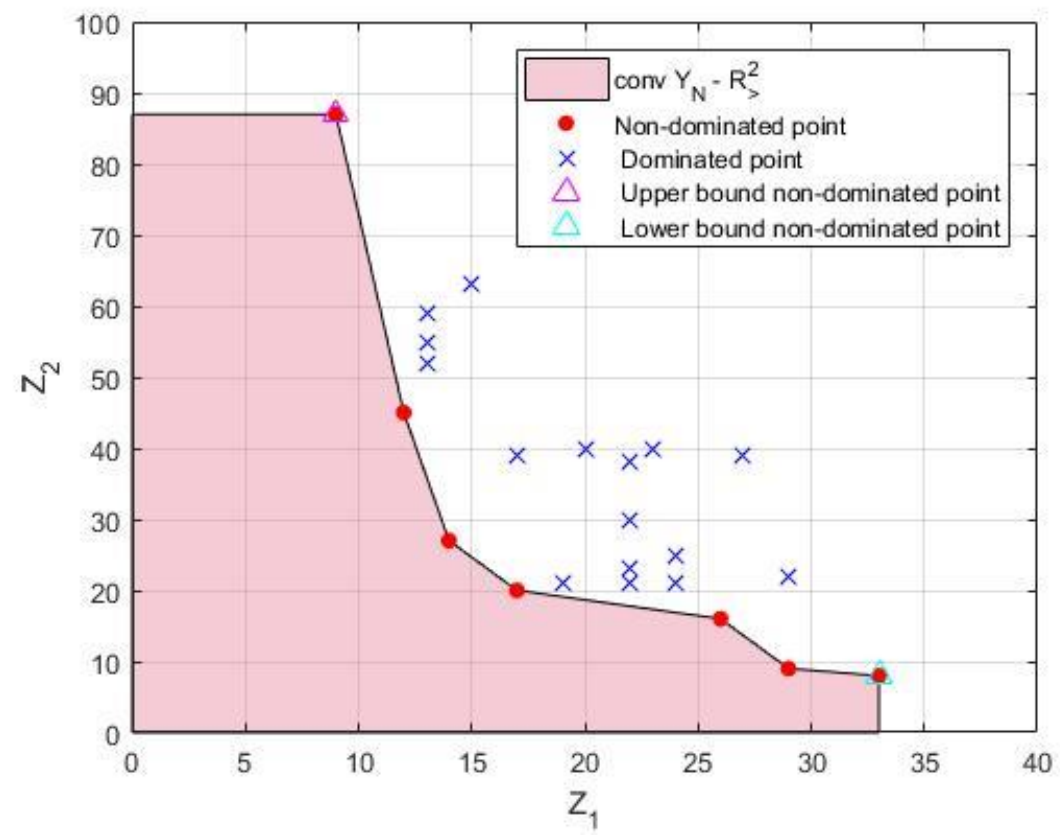

Figure 1. All potential non-dominated points

Table 5. Potential non-dominated points for the first objective function $Z_{1}$

\begin{tabular}{|c|c|c|c|c|}
\hline No. & $Z^{\text {rank }}$ & $Z_{1}^{k}$ & $\left(Z_{1}^{k}, Z_{2 @ Z_{1}^{k}}\right)$ & Potential non-dominated points \\
\hline 1 & $Z_{1}^{\text {opt }}$ & 9 & $(9,87)$ & $(9,87)$ \\
\hline 2 & $Z_{1}^{2}$ & 12 & $(12,45)$ & $(12,45)$ \\
\hline 3 & $Z_{1}^{3}$ & 13 & $(13,59)$ & - \\
\hline 4 & $Z_{1}^{3}$ & 13 & $(13,52)$ & $(13,52)$ \\
\hline 5 & $Z_{1}^{3}$ & 13 & $(13,55)$ & - \\
\hline 6 & $Z_{1}^{4}$ & 14 & $(14,27)$ & $(14,27)$ \\
\hline 7 & $Z_{1}^{5}$ & 15 & $(15,63)$ & $(15,63)$ \\
\hline 8 & $Z_{1}^{6}$ & 17 & $(17,20)$ & $(17,20)$ \\
\hline 9 & $Z_{1}^{6}$ & 17 & $(17,39)$ & - \\
\hline 10 & $Z_{1}^{7}$ & 19 & $(19,21)$ & $(19,21)$ \\
\hline 11 & $Z_{1}^{8}$ & 20 & $(20,40)$ & $(20,40)$ \\
\hline 12 & $Z_{1}^{9}$ & 21 & $(21,70)$ & $(21,70)$ \\
\hline 13 & $Z_{1}^{10}$ & 22 & $(22,30)$ & - \\
\hline 14 & $Z_{1}^{10}$ & 22 & $(22,21)$ & $(22,21)$ \\
\hline 15 & $Z_{1}^{10}$ & 22 & $(22,38)$ & - \\
\hline 16 & $Z_{1}^{10}$ & 22 & $(22,23)$ & - \\
\hline 17 & $\frac{1}{Z_{1}^{11}}$ & 23 & $(23,40)$ & $(23,40)$ \\
\hline 18 & $Z_{1}^{12}$ & 24 & $(24,25)$ & - \\
\hline 19 & $Z_{1}^{12}$ & 24 & $(24,21)$ & $(24,21)$ \\
\hline 20 & $Z_{1}^{13}$ & 26 & $(26,16)$ & $(26,16)$ \\
\hline 21 & $Z_{1}^{14}$ & 27 & $(27,39)$ & $(27,39)$ \\
\hline 22 & $Z_{1}^{15}$ & 29 & $(29,9)$ & $(29,9)$ \\
\hline 23 & $Z_{1}^{15}$ & 29 & $(29,22)$ & - \\
\hline 24 & $Z_{1}^{16}$ & 33 & $(33,8)$ & $(33,8)$ \\
\hline
\end{tabular}


International Journal of Mathematical, Engineering and Management Sciences

Vol. 5, No. 6, 1249-1269, 2020

https://doi.org/10.33889/IJMEMS.2020.5.6.093

Table 6. Potential non-dominated points for the second objective function $Z_{2}$

\begin{tabular}{|c|c|c|c|c|}
\hline No. & $Z^{\text {rank }}$ & $Z_{2}^{k}$ & $\left(Z_{1 @ Z_{2}^{k}}, Z_{2}^{k}\right)$ & Potential non-dominated points \\
\hline 1 & $Z_{2}^{\text {opt }}$ & 8 & $(33,8)$ & $(33,8)$ \\
\hline 2 & $Z_{2}^{2}$ & 9 & $(29,9)$ & $(29,9)$ \\
\hline 3 & $Z_{2}^{3}$ & 16 & $(26,16)$ & $(26,16)$ \\
\hline 4 & $Z_{2}^{4}$ & 20 & $(17,20)$ & $(17,20)$ \\
\hline 5 & $Z_{2}^{5}$ & 21 & $(19,21)$ & $(19,21)$ \\
\hline 6 & $Z_{2}^{5}$ & 21 & $(22,21)$ & - \\
\hline 7 & $Z_{2}^{5}$ & 21 & $(24,21)$ & - \\
\hline 8 & $\frac{2}{Z_{2}^{6}}$ & 22 & $(29,22)$ & $(29,22)$ \\
\hline 9 & $Z_{2}^{7}$ & 23 & $(22,23)$ & $(22,23)$ \\
\hline 10 & $Z_{2}^{8}$ & 25 & $(24,25)$ & $(24,25)$ \\
\hline 11 & $Z_{2}^{9}$ & 27 & $(14,27)$ & $(14,27)$ \\
\hline 12 & $Z_{2}^{10}$ & 30 & $(22,30)$ & $(22,30)$ \\
\hline 13 & $Z_{2}^{11}$ & 38 & $(22,38)$ & $(22,38)$ \\
\hline 14 & $Z_{2}^{12}$ & 39 & $(27,39)$ & - \\
\hline 15 & $Z_{2}^{12}$ & 39 & $(17,39)$ & $(17,39)$ \\
\hline 16 & $Z_{2}^{13}$ & 40 & $(23,40)$ & - \\
\hline 17 & $Z_{2}^{13}$ & 40 & $(20,40)$ & $(20,40)$ \\
\hline 18 & $Z_{2}^{14}$ & 45 & $(12,45)$ & $(12,45)$ \\
\hline 19 & $Z_{2}^{15}$ & 52 & $(13,52)$ & $(13,52)$ \\
\hline 20 & $Z_{2}^{16}$ & 55 & $(13,55)$ & $(13,55)$ \\
\hline 21 & $Z_{2}^{17}$ & 59 & $(13,59)$ & $(13,59)$ \\
\hline 22 & $Z_{2}^{18}$ & 63 & $(15,63)$ & $(15,63)$ \\
\hline 23 & $Z_{2}^{19}$ & 70 & $(21,70)$ & $(21,70)$ \\
\hline 24 & $Z_{2}^{20}$ & 87 & $(9,87)$ & $(9,87)$ \\
\hline
\end{tabular}

Table 7. All non-dominated points for Example 1

\begin{tabular}{|c|c|c|}
\hline No. & $\left(Z_{1}^{\text {rank }}, Z_{2}^{\text {rank }}\right)$ & Non-dominated point \\
\hline 1 & $\left(\mathrm{Z}_{1}^{\text {opt }}, \mathrm{Z}_{2}^{20}\right)$ & $(9,87)$ \\
\hline 2 & $\left(\mathrm{Z}_{1}^{2}, \mathrm{Z}_{2}^{14}\right)$ & $(12,45)$ \\
\hline 3 & $\left(\mathrm{Z}_{1}^{4}, \mathrm{Z}_{2}^{9}\right)$ & $(14,27)$ \\
\hline 4 & $\left(\mathrm{Z}_{1}^{6}, \mathrm{Z}_{2}^{4}\right)$ & $(26,16)$ \\
\hline 5 & $\left(\mathrm{Z}_{1}^{13}, \mathrm{Z}_{2}^{3}\right)$ & $(29,9)$ \\
\hline 6 & $\left(\mathrm{Z}_{1}^{15}, \mathrm{Z}_{2}^{2}\right)$ & $(33,8)$ \\
\hline 7 & $\left(\mathrm{Z}_{1}^{16}, \mathrm{Z}_{2}^{\mathrm{opt}}\right)$ & \\
\hline
\end{tabular}

\section{Example 5}

In order to illustrate the RBSM for a multi-objective situation, the third objective function is added to Example 4,

Let

$$
P_{i, j}^{3}=\left[\begin{array}{llll}
15 & 2 & 5 & 8 \\
3 & 6 & 10 & 11 \\
22 & 6 & 6 & 4 \\
20 & 7 & 3 & 10
\end{array}\right]
$$

- Once again find the optimal solutions in respect of $Z_{1}, Z_{2}$ and $Z_{3}$. Let these optimal solutions be denoted by $Z_{1}^{1}, Z_{2}^{1}$ and $Z_{3}^{1}$, respectively. The values of $Z_{1}^{1}=9, Z_{2}^{1}=8$ are known from example 4 and $Z_{3}^{1}=12$. 
- Find the value of $\left(Z_{2}, Z_{3}\right)$ at the point where $Z_{1}^{1}=9$. This gives first non-dominated points $\left(Z_{1}^{1}, Z_{2 @ Z_{1}^{1}}, Z_{3 @ Z_{1}^{1}}\right)=(9,87,38)$. Similarly, find two more non-dominated points with respect to $Z_{2}^{1}$ and $Z_{3}^{1}$. These points are given by $\left(Z_{1 @ Z_{2}^{1}}, Z_{2}^{1}, Z_{3 @ Z_{2}^{1}}\right)=(33,8,35)$ and $\left(Z_{1 @ Z_{3}^{1}}, Z_{2 @ Z_{3}^{1}}, Z_{3}^{1}\right)=(15,63,12)$, respectively.

- Using the RSM (Kumar et al., 2018) find ranked optimal solutions in respect of $Z_{1}$, denoted by $Z_{1}^{K}$, such that $Z_{1}^{K} \leq \operatorname{Max}\left(Z_{1 @ Z_{2}^{1}}, Z_{1 @ Z_{3}^{1}}\right)$. Similarly, repeat on $Z_{2}$ and $Z_{3}$ such that $Z_{2}^{K} \leq$ $\operatorname{Max}\left(Z_{2 @ Z_{1}^{1}}, Z_{2 @ Z_{3}^{1}}\right)$ and $Z_{3}^{K} \leq \operatorname{Max}\left(Z_{3 @ Z_{1}^{1}}, Z_{3 @ Z_{2}^{1}}\right)$, respectively. $K=2,3, \ldots$.

- $\quad$ For each $Z_{1}^{K}$, find $\left(Z_{2 @ Z_{1}^{K}}, Z_{3 @ Z_{1}^{K}}\right)$ to obtain the potential non-dominated points denoted by $\left(Z_{1}^{K}, Z_{2 @ Z_{1}^{K}}, Z_{3 @ Z_{1}^{K}}\right)$, see Table 9 in the appendix. Similarly, determine the potential nondominated points in respect of $Z_{2}^{K}$ and $Z_{3}^{K}$, which are denoted $\left(Z_{1 @ Z_{2}^{K}}, Z_{2}^{K}, Z_{3 @ Z_{2}^{K}}\right)$ and $\left(Z_{1 @ Z_{3}^{K}}, Z_{2 @ Z_{3}^{K}}, Z_{3}^{K},\right)$, respectively. The Tables 8,9 and 10 give the potential non-dominated points.

- Using definition 6, all potential non-dominated points in Tables 8, 9 and 10 are tested and the set of non- dominated point $Y_{N D}$ are shown in Table 11.

Table 8. Potential non-dominated points for first objective function

\begin{tabular}{|c|c|c|c|c|}
\hline No. & $Z_{1}^{\text {rank }}$ & $Z_{1}^{l_{1}}$ & Potential non-dominated points & Non-dominated points \\
\hline 1 & $Z_{1}^{o p t}$ & 9 & $(9,87,38)$ & $(9,87,38)$ \\
\hline 2 & $Z_{1}^{2}$ & 12 & $(12,45,21)$ & $(12,45,21)$ \\
\hline 3 & $Z_{1}^{3}$ & 13 & $(13,59,20)$ & $(13,59,20)$ \\
\hline 4 & $Z_{1}^{3}$ & 13 & $(13,52,35)$ & - \\
\hline 5 & $Z_{1}^{3}$ & 13 & $(13,55,44)$ & - \\
\hline 6 & $Z_{1}^{4}$ & 14 & $(14,27,24)$ & $(14,27,24)$ \\
\hline 7 & $Z_{1}^{5}$ & 15 & $(15,63,12)$ & $(15,63,12)$ \\
\hline 8 & $Z_{1}^{6}$ & 17 & $(17,20,41)$ & $(17,20,41)$ \\
\hline 9 & $Z_{1}^{6}$ & 17 & $(17,39,39)$ & - \\
\hline 10 & $Z_{1}^{7}$ & 19 & $(19,21,42)$ & - \\
\hline 11 & $Z_{1}^{8}$ & 20 & $(20,40,45)$ & - \\
\hline 12 & $Z_{1}^{9}$ & 21 & $(21,70,39)$ & - \\
\hline 13 & $Z_{1}^{10}$ & 22 & $(22,30,24)$ & - \\
\hline 14 & $Z_{1}^{10}$ & 22 & $(22,21,34)$ & - \\
\hline 15 & $Z_{1}^{10}$ & 22 & $(22,38,43)$ & - \\
\hline 16 & $Z_{1}^{10}$ & 22 & $(22,23,39)$ & $(22,23,39)$ \\
\hline 17 & $Z_{1}^{11}$ & 23 & $(23,40,47)$ & - \\
\hline 18 & $Z_{1}^{12}$ & 24 & $(24,25,36)$ & $(24,25,36)$ \\
\hline 19 & $Z_{1}^{12}$ & 24 & $(24,21,37)$ & $(24,21,37)$ \\
\hline 20 & $Z_{1}^{13}$ & 26 & $(26,16,19)$ & $(26,16,19)$ \\
\hline 21 & $Z_{1}^{14}$ & 27 & $(27,39,28)$ & - \\
\hline 22 & $Z_{1}^{15}$ & 29 & $(29,9,36)$ & $(29,9,36)$ \\
\hline 23 & $Z_{1}^{15}$ & 29 & $(29,22,40)$ & - \\
\hline 24 & $Z_{1}^{16}$ & 33 & $(33,8,35)$ & $(33,8,35)$ \\
\hline
\end{tabular}


International Journal of Mathematical, Engineering and Management Sciences

Vol. 5, No. 6, 1249-1269, 2020

https://doi.org/10.33889/IJMEMS.2020.5.6.093

Table 9. Potential non-dominated points for second objective function

\begin{tabular}{|c|c|c|c|c|}
\hline No. & $Z_{2}^{\text {rank }}$ & $Z_{2}^{l_{2}}$ & Potential non-dominated points & Non-dominated points \\
\hline 1 & $Z_{2}^{\text {opt }}$ & 8 & $(33,8,35)$ & $(33,8,35)$ \\
\hline 2 & $Z_{2}^{2}$ & 9 & $(29,9,36)$ & $(29,9,36)$ \\
\hline 3 & $Z_{2}^{3}$ & 16 & $(26,16,19)$ & $(26,16,19)$ \\
\hline 4 & $Z_{2}^{4}$ & 20 & $(17,20,41)$ & $(17,20,41)$ \\
\hline 5 & $Z_{2}^{5}$ & 21 & $(19,21,42)$ & - \\
\hline 6 & $Z_{2}^{5}$ & 21 & $(22,21,34)$ & - \\
\hline 7 & $Z_{2}^{5}$ & 21 & $(24,21,37)$ & $(24,21,37)$ \\
\hline 8 & $Z_{2}^{6}$ & 22 & $(29,22,40)$ & - \\
\hline 9 & $Z_{2}^{7}$ & 23 & $(22,23,39)$ & $(22,23,39)$ \\
\hline 10 & $Z_{2}^{8}$ & 25 & $(24,25,36)$ & $(24,25,36)$ \\
\hline 11 & $Z_{2}^{9}$ & 27 & $(14,27,24)$ & $(14,27,24)$ \\
\hline 12 & $Z_{2}^{10}$ & 30 & $(22,30,24)$ & - \\
\hline 13 & $Z_{2}^{11}$ & 38 & $(22,38,34)$ & - \\
\hline 14 & $Z_{2}^{12}$ & 39 & $(27,39,28)$ & - \\
\hline 15 & $Z_{2}^{12}$ & 39 & $(17,39,39)$ & - \\
\hline 16 & $Z_{2}^{13}$ & 40 & $(23,40,47)$ & - \\
\hline 17 & $Z_{2}^{13}$ & 40 & $(20,40,45)$ & - \\
\hline 18 & $Z_{2}^{14}$ & 45 & $(12,45,21)$ & $(12,45,21)$ \\
\hline 19 & $Z_{2}^{15}$ & 52 & $(13,52,35)$ & - \\
\hline 20 & $Z_{2}^{16}$ & 55 & $(13,55,44)$ & - \\
\hline 21 & $Z_{2}^{17}$ & 59 & $(13,59,20)$ & $(13,59,20)$ \\
\hline 22 & $Z_{2}^{18}$ & 63 & $(15,63,12)$ & $(15,63,12)$ \\
\hline 23 & $Z_{2}^{19}$ & 70 & $(21,70,39)$ & - \\
\hline 24 & $Z_{2}^{20}$ & 87 & $(9,87,38)$ & $(9,87,38)$ \\
\hline
\end{tabular}

Table 10. Potential non-dominated points for third objective function

\begin{tabular}{|c|c|c|c|c|}
\hline No. & $Z_{3}^{\text {rank }}$ & $Z_{3}^{l_{3}}$ & Potential non-dominated points & Non-dominated points \\
\hline 1 & $Z_{3}^{o p t}$ & 12 & $(15,63,12)$ & $(15,63,12)$ \\
\hline 2 & $Z_{3}^{2}$ & 19 & $(26,16,19)$ & $(26,16,19)$ \\
\hline 3 & $Z_{3}^{3}$ & 20 & $(13,59,20)$ & $(13,59,20)$ \\
\hline 4 & $Z_{3}^{4}$ & 21 & $(12,45,21)$ & $(12,45,21)$ \\
\hline 5 & $Z_{3}^{5}$ & 24 & $(14,27,24)$ & $(14,27,24)$ \\
\hline 6 & $Z_{3}^{5}$ & 24 & $(22,30,24)$ & - \\
\hline 7 & $Z_{3}^{6}$ & 28 & $(27,39,28)$ & - \\
\hline 8 & $Z_{3}^{7}$ & 35 & $(33,8,35)$ & $(33,8,35)$ \\
\hline 9 & $Z_{3}^{7}$ & 35 & $(13,52,35)$ & - \\
\hline 10 & $Z_{3}^{8}$ & 36 & $(29,9,36)$ & $(29,9,36)$ \\
\hline 11 & $Z_{3}^{8}$ & 36 & $(24,25,36)$ & $(24,25,36)$ \\
\hline 12 & $Z_{3}^{9}$ & 37 & $(24,21,37)$ & $(24,21,37)$ \\
\hline 13 & $Z_{3}^{10}$ & 38 & $(9,87,38)$ & $(9,87,38)$ \\
\hline 14 & $Z_{3}^{11}$ & 39 & $(22,23,39)$ & $(22,23,39)$ \\
\hline 15 & $Z_{3}^{11}$ & 39 & $(17,39,39)$ & - \\
\hline 16 & $Z_{3}^{11}$ & 39 & $(21,70,39)$ & - \\
\hline 17 & $Z_{3}^{12}$ & 40 & $(29,22,40)$ & - \\
\hline 18 & $Z_{3}^{13}$ & 41 & $(17,20,41)$ & $(17,20,41)$ \\
\hline
\end{tabular}


International Journal of Mathematical, Engineering and Management Sciences

Vol. 5, No. 6, 1249-1269, 2020

https://doi.org/10.33889/IJMEMS.2020.5.6.093

Table 11. All non-dominated points

\begin{tabular}{|c|c|c|}
\hline No. & $\left(Z_{1}^{\text {rank }}, Z_{2}^{\text {rank }}, Z_{3}^{\text {rank }}\right)$ & Non-dominated point \\
\hline 1 & $\left(Z_{1}^{\text {ot }}, Z_{2}^{20}, Z_{3}^{10}\right)$ & $(9,87,38)$ \\
\hline 2 & $\left(Z_{1}^{2}, Z_{2}^{14}, Z_{3}^{4}\right)$ & $(12,45,21)$ \\
\hline 3 & $\left(Z_{1}^{4}, Z_{2}^{17}, Z_{3}^{3}\right)$ & $(13,59,20)$ \\
\hline 4 & $\left(Z_{1}^{4}, Z_{2}^{9}, Z_{3}^{5}\right)$ & $(14,27,24)$ \\
\hline 5 & $\left(Z_{1}^{5}, Z_{2}^{18}, Z_{3}^{\text {opt }}\right)$ & $(15,63,12)$ \\
\hline 6 & $\left(Z_{1}^{6}, Z_{2}^{4}, Z_{3}^{13}\right)$ & $(22,23,41)$ \\
\hline 7 & $\left(Z_{1}^{10}, Z_{2}^{10}, Z_{3}^{11}\right)$ & $(24,25,36)$ \\
\hline 8 & $\left(Z_{1}^{11}, Z_{2}^{8}, Z_{3}^{8}\right)$ & $(24,21,37)$ \\
\hline 9 & $\left(Z_{1}^{12}, Z_{2}^{5}, Z_{3}^{9}\right)$ & $(26,16,19)$ \\
\hline 10 & $\left(Z_{1}^{13}, Z_{2}^{3}, Z_{3}^{2}\right)$ & $(29,9,36)$ \\
\hline 11 & $\left(Z_{1}^{11}, Z_{2}^{2}, Z_{3}^{8}\right)$ & $(33,8,35)$ \\
\hline 12 & $\left(Z_{1}^{16}, Z_{2}^{\text {opt }}, Z_{3}^{7}\right)$ & \\
\hline
\end{tabular}

\section{Conclusion and Future Study}

The importance of an optimal solution in single-objective problems is well known, however, in this paper, a need for ranked-optimal solutions has also been established. This paper also points out that ranked-optimal solutions are computationally demanding and there is a need to establish more methods capable to find efficiently ranked-optimal solutions. In this paper, a rank-optimal solution method has been established to find non-dominated point set for a multi-objective problem.

Future studies will consider the application of rank-optimal solution method for other models such as Knapsack and transportation.

Conflict of Interest

The authors declare that there is no conflict for this publication.

Acknowledgements

The authors are grateful to the referees for their constructive suggestions and helpful comments.

\section{References}

Al-Hasani, A., Al-Rabeeah, M., Kumar, S., \& Eberhard, A. (2019). Finding all non-dominated points for a bi-objective generalized assignment problem. In Journal of Physics: Conference Series (Vol. 1218, No. 1, p. 012004). IOP Publishing.

Al-Rabeeah, M., Al-Hasani, A., Kumar, S., \& Eberhard, A. (2020). Enhancement on the improved recursive method for multi-objective integer programming problem. In International Conference on Mathematics: Pure, Applied and Computation Mathematics for Better Future Life ICoMPAC 2019(Indonesia).

Al-Rabeeah, M., Kumar, S., Al-Hasani, A., Munapo, E., \& Eberhard, A. (2019). Bi-objective integer programming analysis based on the characteristic equation. International Journal of System Assurance Engineering and Management, 10(5), 937-944. 
International Journal of Mathematical, Engineering and Management Sciences

Vol. 5, No. 6, 1249-1269, 2020

https://doi.org/10.33889/IJMEMS.2020.5.6.093

Boland, N., Charkhgard, H., \& Savelsbergh, M. (2015). A criterion space search algorithm for bi-objective integer programming: the balanced box method. INFORMS Journal on Computing, 27(4),735-754.

Boland, N., Charkhgard, H., \& Savelsbergh, M. (2016). The L-shape search method for triobjective integer programming. Mathematical Programming Computation, 8(2), 217-251.

Ehrgott, M. \& Gandibleux, X. (2000). A survey and annotated bibliography of multi-objective combinatorial optimization. OR-Spektrum, 22(4), 425-460.

Ehrgott, M. (2005). Multicriteria optimization. Springer Science \& Business Media, New York.

Greco, S., Figueira, J., \& Ehrgott, M. (2016). Multiple criteria decision analysis. Springer, New York.

Haimes, Y. (1971). On a bicriterion formulation of the problems of integrated system identification and system optimization. IEEE Transactions on Systems, Man, And Cybernetics, 1(3), 296-297.

Kirlik, G. \& Sayın, S. (2014). A new algorithm for generating all nondominated solutions of multi-objective discrete optimization problems. European Journal of Operational Research, 232(3), 479-488.

Kumar, S. \& Munapo, E. (2012). Some lateral ideas and their applications for developing new solution procedures for a pure integer programming model, Keynote address. In Mariappan, Srinivasan and Amritraj (eds), Proceedings of Herbal International Conference on applications of mathematics and statistics for intelligent solutions through Mathematics and Statistics, Excel India Publishers, pp 13-21.

Kumar, S., Al-Hasani, A., Al-Rabeeah, M., \& Eberhard, A. (2018). A random search method for finding ' $k$ $\geq 2$ ' number of ranked optimal solution to an assignment problem. In http://www.optimizationonline.org/DB_HTML/2018/11/6942.html.

Kumar, S., Luhandjula, M.K., Munapo, E., \& Jones, B.B. (2010). Fifty years of integer programming: a review of solution approaches. Asia Pacific Business Review, 6(2), 5-15.

Kumar, S., Munapo, E., \& Jones, B.C. (2007). An integer equation controlled descending path to a protean pure integer program. Indian Journal of Mathematics, 49(2), 211-237.

Laumanns, M., Thiele, L., \& Zitzler, E. (2006). An efficient, adaptive parameter variation scheme for metaheuristics based on the epsilon-constraint method. European Journal of Operational Research, 169(3):932-942.

Murty, K.G. (1968). An algorithm for ranking all the assignments in order of increasing costs. Operations research, 16(3), 682-687.

Özlen, M., \& Azizoğlu, M. (2009). Multi-objective integer programming: a general approach for generating all non-dominated solutions. European Journal of Operational Research, 199(1), 25-35.

Özlen, M., Burton, B.A., \& MacRae, C.A. (2014). Multi-objective integer programming: an improved recursive algorithm. Journal of Optimization Theory and Applications, 160(2), 470-482.

Przybylski, A., Gandibleux, X., \& Ehrgott, M. (2008). Two phase algorithms for the bi-objective assignment problem. European Journal of Operational Research, 185(2), 509-533.

Przybylski, A., Gandibleux, X., \& Ehrgott, M. (2010a). A recursive algorithm for finding all nondominated extreme points in the outcome set of a multi-objective integer programme. INFORMS Journal on Computing, 22(3), 371-386.

Przybylski, A., Gandibleux, X., \& Ehrgott, M. (2010b). A two-phase method for multi-objective integer programming and its application to the assignment problem with three objectives. Discrete Optimization, 7(3), 149-165.

Steuer, R.E. (1986). Multiple criteria optimization: theory, computation, and applications. John Wiley. New York. 
International Journal of Mathematical, Engineering and Management Sciences

Vol. 5, No. 6, 1249-1269, 2020

https://doi.org/10.33889/IJMEMS.2020.5.6.093

Sylva, J., \& Crema, A. (2004). A method for finding the set of non-dominated vectors for multiple objective integer linear programs. European Journal of Operational Research, 158(1), 46-55.

Sylva, J., \& Crema, A. (2007). A method for finding well-dispersed subsets of non-dominated vectors for multiple objective mixed integer linear programs. European Journal of Operational Research, 180(3), 1011-1027.

Teghem, J., Tuyttens, D., \& Ulungu, E.L. (2000). An interactive heuristic method for multi-objective combinatorial optimization. Computers \& Operations Research, 27(7-8), 621-634.

Tuyttens, D., Teghem, J., Fortemps, P., \& Van Nieuwenhuyze, K. (2000). Performance of the mosa method for the bicriteria assignment problem. Journal of Heuristics, 6(3), 295-310.

Ulungu, E., \& Teghem, J. (1995). The two phases method: an efficient procedure to solve bi-objective combinatorial optimization problems. Foundations of Computing and Decision Sciences, 20(2), 149165. 